\title{
In honor of the 65th birthday of Professor Zhi- dong Bai
}

\author{
By HU FeiFang \& CHEN ZeHua, Guest Editors
}

We are thankful to Science in China Series A: Mathematics for the publication of this special issue in honor of Professor Zhidong Bai of his 65th birthday. Professor Bai is a leading statistician in the world. He has made indelible contributions in a broad range of statistical areas. He was one of the first $18 \mathrm{PhDs}$ in China whose degrees were awarded by the State Council in 1982. Professor Bai represents a special generation who experienced the most difficult period in China but never gave up their quest for science and eventually excelled in the world arena. This special issue is not only to honor Professor Bai for his great achievements but also to inspire the young generation to devote themselves to the promotion of Statistics in China as China enters into a great new era. Professor Bai has a legendary life. He was adopted by a poor peasant family at birth. He spent his childhood during the Chinese resistance war against Japan. He had an incomplete elementary education under extremely backward conditions. Yet he managed to enter the University of Science and Technology of China (USTC) in 1963. After graduation from USTC, he worked as a truck driver's team leader and was completely detached from the academic for ten years during the Cultural Revolution. However, he got admitted into the graduate program of USTC in 1978 and renewed his academic career. After his graduation he established a collaborative relationship with Professor C. R. Rao, one of the most famous statisticians in modern history of Statistics. He soon had his academic power felt. He was elected as a Fellow of the Third World Academy of Sciences in 1989 and a Fellow of the Institute of Mathematical Statistics in 1990.

Professor Bai has worked at University of Pittsburgh, Temple University, Sun-Yat-Sen University at Taiwan, National University of Singapore and Northeast China Normal University. His research areas include random matrix theory, Edgeworth expansion, M-estimation, model selection, adaptive design in clinical trials, applied probability in algorithms, small area estimation, time series, and so on. He has published three monographs and over 160 research papers and left his important marks in all above mentioned areas.

To honor Professor Bai on the occasion of his 65th birthday, a one-day conference was held at the National University of Singapore on 20 July, 2008. Over 100 statisticians and students from all over the world had attended this one-day workshop. Eleven professors had presented their research work. They are: G. J. Babu (Pennsylvania State University), Arup Bose (Indian Statistics Institute), Siuhung Cheung (The Chinese University of Hong Kong), Xuming He (University of Illinois at Urbana-Champaign), Feifang Hu (University of Virginia), WongWing Keung (Hong Kong Baptist University), Xiaoli Meng (Harvard University ), Qiman Shao (Hong Kong University of Science and Technology), Ben-Chang Shia (Fu Jen Catholic University), Yuehua Wu (York University) and Zhenlin Yang (Singapore Management University). Most of papers in this special issue are contributed by some of the participants of the conference.

This special issue consists of ten research papers from a number of active areas of statisti- 
cal research. C. R. Rao (Penn State University) and Yuehua Wu (York University) consider constrained M-estimation in a general multivariate linear regression model. Arup Bose (Indian Statistical Institute) studies the asymptotic distribution of the $L_{1}$ regression estimate. Jin-Ting Zhang and Jinfeng Xu (National University of Singapore) consider the $k$-sample Behrens-Fisher problem for high-dimensional data. Zehua Chen (National University of Singapore) and Jiahua Chen (University of British Columbia) propose to use tournament screening cum EBIC for feature selection in high dimensional spaces. Qiman Shao (Hong Kong University of Science and Technology) and Rongmao Zhang (Zhejiang University) derive a general result on the limiting distribution of a non-central studentized statistic. Lixin Zhang (Zhejiang University) and Feifang Hu (University of Virginia) study the Gaussian approximation for multi-color generalized Friedman's urn model. Yu Hu, Xuming He (University of Illinois at Urbana-Champaign), Jian Tao and NingZhong Shi (North Normal University) use the functional principal component analysis to model and predict growth in children. B. Y. Jing, X. B. Kong, Z. Liu (Hong Kong University of Science and Technology) and B. Zhang (Renmin University of China) apply stochastic regression to hedging in finance. D. Kundu (Indian Institute of Technology Kanpur) and B. Pradhan (Indian Statistical Institute) consider Bayesian inference for generalized exponential distributions. We are grateful to the authors and anonymous reviewers who contributed their time and effort to this special issue. Special thanks go to Ruiyan Zhang for her coordination of this special issue.

\section{Guest Editor Biography}

Feifang $\mathrm{Hu}$ is Professor of Statistics at University of Virginia. He is an elected Fellow of the Institute of Mathematical Statistics (IMS) and Fellow of the American Statistical Association (ASA). He serves on the editorial boards of the Annals of Statistics and Statistics and Its Interface. For more information, please visit his website at www.stat.virginia.edu/hu.html.

Zehua Chen is Professor of Statistics at National University of Singapore. He serves on the editorial boards of Journal of Statistical Planning and Inference and Journal of Nonparametrics. For more information, please visit his website at http://www.stat.nus.edu.sg/stachenz/. 